\section{Estrategias de marketing en el canal intermedio y posicionamiento para el sector de empresas comercializadoras de GPS en el Perú periodo 2018 - 2020}

\author{
Marketing Strategies in the intermediate channel and \\ positioning for the sector of GPS marketing companies in \\ Perú period 2018 - 2020
}

\begin{abstract}
RESUMEN
El canal intermedio representa un alto nivel de rentabilidad para las empresas comercializadoras de GPS, debido a sus alianzas estratégicas con las aseguradoras para obtener la base de datos de los clientes que requieren la instalación del GPS de manera obligatoria antes de recibir sus autos nuevos y que serán derivados de manera automática a sus empresas. Por lo cual, la presente investigación tiene como objetivo comprobar la relación que existe entre las estrategias de marketing del canal intermedio y el posicionamiento de las empresas que brindan servicio de GPS en todo el Perú para el periodo 2018 - 2020. El enfoque ha sido cuantitativo; diseño no experimental, descriptivo, correlacional y longitudinal, siendo el cuestionario, el instrumento de recopilación de datos que fue aplicado en una muestra de 168 clientes con servicio de GPS. Demostrando con los resultados obtenidos, la existencia de una relación de estrategias de marketing orientada al canal intermedio y el posicionamiento de las empresas que comercializan GPS en Perú, con un valor de Chi cuadrado de 334,868 y un nivel de significación de 0.001 , siendo esta muy alta. Por tanto, la creación de alianzas estratégicas es una necesidad para obtener una mayor rentabilidad en las aseguradoras vehiculares y el mejor posicionamiento de las empresas de GPS.
\end{abstract}

Palabras Clave: Canal Intermedio; posicionamiento empresarial; estrategias de marketing; GPS.

\begin{abstract}
The intermediate channel represents a high level of profitability for GPS marketing companies, due to their strategic alliances with insurers to obtain the database of customers who require the installation of GPS on a mandatory basis before receiving their new cars and that they will be automatically referred to your companies. Therefore, the present research aims to verify the relationship that exists between the marketing strategies of the intermediate channel and the positioning of the companies that provide GPS services throughout Peru for the period 2018 - 2020. The approach has been quantitative; non-experimental, descriptive, correla-
\end{abstract}

\section{Augusto Gerardo León \\ Ratto}

20020858@lamolina.edu.pe

Universidad Nacional Agraria La Molina. Lima, Perú

Presentado: 02/12/2020 - Aceptado: 09/08/2021 - Publicado: 23/12/2021

(C) Los autores. Este artículo es publicado por la revista Gestión en el Tercer Milenio de la Facultad de Ciencias Administrativas Universidad Nacional Mayor de San Marcos. Este es un artículo de acceso abierto, distribuido bajo los términos de la licencia Creative Commons Atribución 4.0 Internacional (CC BY 4.0) [https://creativecommons.org/licenses/by/4.0/deed.es] que permite el uso, distribución y reproducción en cualquier medio, siempre que la obra original sea debidamente citada de su fuente original. 
tional and longitudinal design, the questionnaire being the data collection instrument that was applied in a sample of 168 clients with GPS service. Demonstrating with the results obtained, the existence of a relationship of marketing strategies oriented to the intermediate channel and the positioning of the companies that market GPS in Peru, with a Chi square value of 334.868 and a significance level of 0.001 , this being very high. Therefore, the creation of strategic alliances is a necessity to obtain greater profitability in vehicle insurers and the better positioning of GPS companies.

Keywords: Intermediate Channel; business positioning; marketing strategies; GPS.

\section{INTRODUCCIÓN}

Según el Ministerio de Transporte y Comunicaciones (2017), cada cinco minutos en el Perú ocurre un accidente vehicular, por cada hora transcurrida se dan dos personas discapacitadas, por cada tres horas una persona fallece en un accidente de autos y el costo anual que representa para el Perú los accidentes vehiculares es de 8,475 millones de soles. Por otro lado, la Asociación Automotriz del Perú (2017) nos denota que un total de 163,668 vehículos, entre autos y camionetas ligeras, ingresó al parque automotor en el año 2017, los cuales contaron de manera obligatoria con un Sistema de Posicionamiento Global (GPS; en inglés, Global Positioning System) al momento de su venta y la facturación promedio de este es de US\$36'006,960 dólares, considerando un precio estándar de US\$220 por dispositivo GPS instalado.

Cabe señalar, que las empresas comercializadoras de GPS están formadas por tres canales en ventas: Canal Directo (el usuario final que compra de manera independiente un GPS para su auto), Canal intermedio (son las aseguradoras de los vehículos que obligan el uso de GPS y derivan la información de los clientes que adquieren autos nuevos a las empresas comercializadoras de GPS) y por último el Canal corporativo (son las flotas de vehículos que requieren GPS para sus operaciones comerciales). Por tanto, para fines de esta investigación, se desarrolló el estudio en el canal intermedio y sus estrategias de marketing correspondientes.

Como canal intermedio, actualmente las aseguradoras vehiculares necesitan contar con una tecnología que brinde un reporte actualizado sobre la forma de manejo de sus clientes, para que se puedan tomar las medidas correspondientes al identificar un estilo de conducción agresivo. Esto último, se sustenta con el estudio realizado por la empresa Tracklink Octo (Gestión, 2016) que afirma que el estilo de conducción en el Perú es calificado de agresivo y se evidencia en el alto costo de las pólizas vehiculares. Debido a la elevada siniestralidad vehicular, las empresas aseguradoras generan pólizas para incentivar y reforzar los buenos hábitos de manejo.

Es importante mencionar que el canal intermedio comenzó a crecer de manera rápida, debido a la exigencia por parte de las aseguradoras vehiculares a sus clientes de contar con GPS por un periodo mínimo de 2 años al adquirir un vehículo nuevo. Con la aplicación de las estrategias de marketing para el canal intermedio, se otorga a las aseguradoras vehiculares el apoyo tecnológico por parte de las empresas comercializadoras de GPS, para que puedan informar a sus clientes sobre su forma de manejo y poder disminuir la tasa de siniestralidad actual.

Las empresas comercializadoras de GPS obtienen la lista de clientes que están a la espera de la instalación inmediata de GPS y que es brindada directamente por parte de las aseguradoras vehiculares que gestionaron el trato directo con el cliente. Es decir, ambas partes se beneficiarían: las aseguradoras disminuirían el nivel de siniestralidad por contar con la información tecnológica del GPS con el estilo de conducción de sus clientes, viéndose reflejado en una mayor rentabilidad; mientras que las empresas comercializadoras de GPS podrían mejorar su posicionamiento en el corto plazo, por el mayor volumen de ventas realizadas en el canal intermedio.

En el contexto actual, el canal intermedio representa un alto nivel de rentabilidad para 
las empresas comercializadoras de GPS, debido a que no invertirán esfuerzos en la búsqueda de nuevos clientes, sino que gracias a las alianzas estratégicas con las aseguradoras lograrán obtener la base de datos actualizadas de todos los clientes que requieren instalar GPS de manera obligatoria y que serán derivados de manera automática a sus empresas y puedan realizar la instalación del servicio de GPS antes que el cliente reciba su auto nuevo. Por lo anterior, se identifica el siguiente problema de investigación: ¿Las estrategias de marketing orientadas al canal intermedio influyen en el posicionamiento del sector de empresas comercializadoras de GPS en el Perú, durante el periodo 20182020 ? El objetivo general de investigación es determinar si las estrategias de marketing, orientadas al canal intermedio influyen en el posicionamiento del sector de empresas comercializadoras de GPS en el Perú, durante el periodo 2018-2020, y la hipótesis general es: Estrategias de marketing, orientadas al canal intermedio influyen de manera significativa en el posicionamiento del sector de empresas comercializadoras de GPS en el Perú, durante el Periodo 2018-2020.

\section{MÉTODOS}

La investigación fue de tipo aplicada, con enfoque cuantitativo y de método exploratorio; diseño no experimental, descriptivo, correlacional y longitudinal para demostrar la relación que existente entre las variables estrategias de marketing y posicionamiento. La población de estudio fue representada por clientes que realizaron la contratación de un seguro vehicular (La Positiva, Rímac, Pacífico, Mapfre y HDI) al momento de la compra de su auto nuevo en el año 2017.

Con el propósito de obtener datos que respalden el nivel de relación entre las variables, se realizó el cálculo estadístico para determinar la muestra de la investigación. La muestra obtenida fue de 168 personas, las cuales realizaron la compra de un auto nuevo con la instalación del servicio de GPS.

Para los fines de la investigación se consideró las referencias de Kotler y Amstrong (2007) quienes utilizaron siete dimensiones propuestas y aplicadas en la variable independiente Estrategias de Marketing, las cuales son:
Reducción de costos, Creación de experiencia única al consumidor; Reinvención del modelo de negocio, Calidad del producto, Centralización en nichos de mercado, Innovación y la Perfección en el diseño del producto. Con respecto a la variable dependiente Posicionamiento plantearon tres dimensiones: Imagen, Producto y Competencia.

De acuerdo con las variables y dimensiones de referencia, se elaboró el cuestionario, el cual fue aplicado a 168 encuestados a nivel Perú. Posteriormente, se sistematizó los datos obtenidos y se analizó mediante la prueba de Chi cuadrado de Pearson para poder encontrar si existe nivel significativo de relación entre las estrategias de marketing orientadas al canal intermedio y el posicionamiento de las empresas comercializadoras de GPS.

Para la realización de la encuesta realizada se consideraron personas que tienen de 25 años a más, debido a que es la edad inicial en la que un cliente compra su primer auto nuevo. La población de estudio son los clientes que contrataron el primer año el seguro del auto, en donde se consideró las cinco principales aseguradoras: Rímac, Pacífico, La Positiva, Mapfre y HDI.

Para los fines de la encuesta se desarrollaron 50 preguntas, siendo 35 preguntas correspondiente a la variable independiente Estrategias de Marketing, que está formado por siete dimensiones con cinco preguntas cada una y se desarrollaron 15 preguntas de la variable dependiente Posicionamiento, que está formado por tres dimensiones y con cinco preguntas cada una.

\section{RESULTADOS}

Del uso del método de Chi cuadrado para poder obtener la regla de decisión y la clasificación de: Malo, Regular, Bueno y Muy bueno por parte de los 168 clientes encuestados, según indica la Tabla 1, los usuarios que cuentan con GPS en sus vehículos (168), el 69.6\% menciona que las estrategias de marketing corresponden a una selección muy bueno (117), siendo el 97.4\% un nivel muy bueno de posicionamiento (38), concentrándose aquí la mayoría de las respuestas.

En ese sentido, cuando se realizó el cálculo del Chi cuadrado, según Tabla 2, nos brindó un 
Tabla 1

Dimensión de Estrategias de Marketing / Posicionamiento

\begin{tabular}{|c|c|c|c|c|c|c|}
\hline & & \multicolumn{4}{|c|}{ Posicionamiento } & \multirow{2}{*}{ Total } \\
\hline & & Malo & Regular & Bueno & Muy Bueno & \\
\hline \multirow{8}{*}{ Estrategia de Marketing } & \multirow{2}{*}{ Malo } & 7 & 0 & 0 & 0 & 7 \\
\hline & & $100,0 \%$ & $0,0 \%$ & $0,0 \%$ & $0,0 \%$ & $4,2 \%$ \\
\hline & \multirow{2}{*}{ Regular } & 0 & 13 & 0 & 0 & 13 \\
\hline & & $0,0 \%$ & $92,9 \%$ & $0,0 \%$ & $0,0 \%$ & $7,7 \%$ \\
\hline & \multirow{2}{*}{ Bueno } & 0 & 1 & 29 & 1 & 31 \\
\hline & & $0,0 \%$ & $7,1 \%$ & $26,9 \%$ & $2,6 \%$ & $18,5 \%$ \\
\hline & \multirow{2}{*}{ Muy bueno } & 0 & 0 & 79 & 38 & 117 \\
\hline & & $0,0 \%$ & $0,0 \%$ & $73,1 \%$ & $97,4 \%$ & $69,6 \%$ \\
\hline \multirow{2}{*}{ Total } & & 7 & 14 & 108 & 39 & 168 \\
\hline & & $100,0 \%$ & $100,0 \%$ & $100,0 \%$ & $100,0 \%$ & $100,0 \%$ \\
\hline
\end{tabular}

Nota. Elaboración Propia.

Tabla 2

Pruebas de Chi-cuadrado

\begin{tabular}{lccc}
\hline & Valor & gl & $\begin{array}{c}\text { Sig. asintótica } \\
\text { (2 caras) }\end{array}$ \\
\hline Chi-cuadrado de Pearson & 334,868 & 9 &, 000 \\
Razón de verosimilitud & 158,294 & 9 &, 000 \\
Asociación lineal por lineal & 101,271 & 1 &, 000 \\
$\mathrm{~N}^{\circ}$ de casos válidos & 168 & & \\
\hline
\end{tabular}

Nota. Elaboración Propia.

valor de 334,868; que además contaba con un nivel de significación calculado de 0.001 , lo que indica que existe una alta diferenciación muy significativa entre la variable Posicionamiento y la de estrategias de marketing.

Luego se procedió a realizar las pruebas de Chi cuadrado a las siete dimensiones de la variable independiente Estrategias de Marketing, según Kotler y Amstrong (2007): Costos Reducidos, Experiencia única, Reinvención del modelo de negocio, Calidad máxima del producto, Nicho de Mercado, Innovación, Mejor Diseño e inmediatamente su comparación con la variable Posicionamiento, para obtener los siguientes resultados:

La dimensión Costos Reducidos muestra que el $42.9 \%$ de encuestados considera que los costos reducidos se encuentran en la categoría de Bueno y el 63\% se encuentra calificado con un nivel bueno con respecto al posicionamiento. Al realizar la prueba de Chi cuadrado se concluyó que existe una alta relación muy significativa entre la variable Posicionamiento y la dimensión Costos reducidos.

La dimensión Experiencia única al consumidor muestra que el $38.7 \%$ de encuestados considera que la experiencia única al consumidor se encuentra en la categoría de Muy Bueno y el 97.4\% se encuentra calificado con un nivel muy bueno con respecto al posicionamiento. Al realizar la prueba de Chi cuadrado se concluyó que existe una alta relación muy significativa entre la variable Posicionamiento y la dimensión EXperiencia única al consumidor.

La dimensión Reinvención del modelo de negocio muestra que el $49.4 \%$ de encuestados considera que la reinvención del modelo de negocio se encuentra en la categoría de Muy Bueno y el $75 \%$ se encuentra calificado con un nivel bueno con respecto al posicionamiento. Al realizar la prueba de Chi cuadrado se concluyó que existe una alta relación muy significativa entre la variable Posicionamiento y la dimensión Reinvención del modelo de negocio. 
La dimensión Calidad del producto muestra que el $45.8 \%$ de encuestados considera que la calidad del producto se encuentra en la categoría de Bueno y el $69.4 \%$ se encuentra calificado con un nivel bueno con respecto al posicionamiento. Al realizar la prueba de Chi cuadrado se concluyó que existe una alta relación muy significativa entre la variable Posicionamiento y la dimensión Calidad máxima del producto.

La dimensión Nicho de mercado muestra que el $38.1 \%$ de encuestados considera que centrarse en un nicho de mercado se encuentran en la categoría de Bueno y el $75.9 \%$ se encuentra calificado con un nivel bueno con respecto al posicionamiento. $\mathrm{Al}$ realizar la prueba de $C h i$ cuadrado se concluyó que existe una alta relación muy significativa entre la variable Posicionamiento y la dimensión Nicho de mercado.

La dimensión Innovación muestra que el $45.8 \%$ de encuestados considera que la innovación se encuentra en la categoría de Bueno y el $69.4 \%$ se encuentra calificado con un nivel bueno con respecto al posicionamiento. $\mathrm{Al}$ realizar la prueba de Chi cuadrado se concluyó que existe una alta relación muy significativa entre la variable Posicionamiento y la dimensión Innovación.

La dimensión Estrategias de diseño muestra que el $61.3 \%$ de encuestados considera que las estrategias de diseño se encuentran en la categoría de Bueno y el $94.4 \%$ se encuentra calificado con un nivel bueno con respecto al posicionamiento. Al realizar la prueba de Chi cuadrado se concluyó que existe una alta relación muy significativa con respecto a la variable Posicionamiento y la dimensión Estrategias de diseño.

\section{DISCUSIÓN}

Lograr el desarrollo de estrategias de marketing en el canal intermedio con la finalidad de mejorar el nivel de posicionamiento de las empresas comercializadoras de GPS en el Perú es muy importante para la expansión de la cartera de clientes en el mercado peruano. Por lo cual, la creación de alianzas estratégicas es una necesidad para obtener una mayor rentabilidad en las aseguradoras vehiculares y empresas de GPS.
El presente estudio, ha identificado que las empresas comercializadoras de GPS cuentan con tres canales de ventas: Directo, Intermedio y Corporativo; y para fines de nuestra investigación se eligió el canal intermedio, debido a que actualmente es el medio de mayor expansión y rentabilidad para las empresas comercializadoras de GPS con las aseguradoras vehiculares.

Lovelock y Wirts (2009), menciona que la estrategia basada en el posicionamiento, considera tomar un lugar distinto para la empresa y las ofertas que pueda obtener de sus diversos productos ofrecidos. Mientras que McCarthy y Perreault (2000), afirman que el concepto del posicionamiento se interpreta como el concepto que tienen los consumidores de las diversas marcas que pueden tener acceso en el mercado. Por otro lado, Lamb et al. (2017) manifiestan que el posicionamiento afecta de una manera directa sobre los clientes que tienen la percepción de la marca, y para fines del presente estudio el canal intermedio es reflejado en 05 marcas de aseguradoras vehiculares, que manejan el mercado actualmente: Rímac, Pacífico, Mapfre, La Positiva y HDI.

Según Keller y Kotler (2006) en su libro Dirección de Marketing indican que el marketing trae como consecuencia que en muy pocos años, sobre todo en los sectores con mucha competencia, la orientación hacia los consumidores se convertirá en una exigencia de base para cualquier empresa.

En el trabajo de investigación por Cubas et al (2015) realizaron un plan de marketing que logró incrementar el Market Share de la empresa Entel, enfocado en el mercado Millenials y dejando análisis estadístico de la rentabilidad alcanzada

Bocanegra (2012), menciona el desarrollo de una aplicación web para el monitoreo de vehículos con dispositivos GPS que comercializa una empresa de telecomunicaciones siendo el punto de partida para mejoras en las aplicaciones existentes de rastreo vehiculares

El estudio evidenciado por Rivas (2015) nos indica que el estilo de conducción en el Perú se caracteriza por ser agresivo, y se ve reflejado en el número de accidentes diarios que se reporta por el MTC. Los malos hábitos 
de conducción y el no respetar las normas de tránsito son una constante en el país.

Por otro lado, el estudio evidenciado en Gestión (2016) deja claro que Perú se encuentra calificado como un país con un estilo muy agresivo en la conducción vehicular, motivo por el cual demanda servicios de calidad para su parque automotor, como el requerimiento básico de GPS en autos nuevos. Actualmente, el control vehicular a través de GPS brinda al cliente la percepción de seguridad mientras conduce, debido a que estos dispositivos cuentan actualmente no solo con ubicación del vehículo, sino con asistencia en caso de robo o alerta en caso de un siniestro, reduciendo el nivel de mortalidad o pérdidas.

El estudio evidenciado en Gestión (2017) nos indica la estrategia que realiza la aseguradora Pacífico, con la finalidad de reducir en $10 \%$ el número de accidentes del país, a través de la creación del aplicativo GPS Smart. El aplicativo tiene la función de medir el estilo de conducción de los clientes midiendo aceleraciones bruscas, frenadas bruscas y aceleraciones bruscas con algoritmos en el sistema.

El artículo del diario La República (2017) nos indica la estrategia realizada por la aseguradora Pacífico con la utilización del aplicativo GPS Smart se canalizaría en el Plan Manejo Bacán, que busca premiar a los conductores que obtengan las mejores calificaciones en su estilo de conducción.

El artículo de diario Perú21 (2017) nos indica la Manejo Bacán realizada por la aseguradora Pacífico y nos explica que dentro de un rango de 0 a 10 de calificación en el estilo de conducción, el $60 \%$ se encuentra con una nota inferior a siete, lo que demuestra el estilo agresivo de conducción en el Perú.

El método de investigación se desarrolló en una muestra de 168 clientes, aplicando un cuestionario de un total de 50 preguntas, del conjunto cabe destacar tres respuestas: Reducción del precio en el GPS, Experiencias del consumidor y Expectativas cubiertas de los clientes sobre sus planes de GPS. De los cuales se detalla a continuación: Sobre la reducción del precio en el GPS se obtuvo que 148 personas (88.1\%) indicaron que es significativo la reducción del precio del GPS y en referencia, Merino y Espino (2009) señalan que se debe aprovechar la ventaja del precio para mejorar el posicionamiento de las empresas. Sobre la realización de encuestas para conocer las experiencias del consumidor, 149 personas (88.7\%) indicaron que es significativo que se realice las encuestas para obtener las experiencias del consumidor, en el sector de GPS y al respecto, Lamb et al. (2017) afirman que el monitoreo constante hacia los clientes mediante encuestas y elaboradas estrategias de marketing va a generar que se puedan tomar acciones correctivas a tiempo frente a cualquier inconveniente que pueda generarse. Sobre la realización de encuestas para conocer las expectativas cubiertas de los clientes que tienen servicio GPS, 148 personas (88.1\%) indicaron que el servicio de GPS cubre sus expectativas y al respecto de ello, Lambin et al. (2009), destacan la importancia de la diferenciación del producto del resto de sus competidores. Es decir, si las empresas comercializadoras de GPS ya cuentan actualmente con gran aceptación del mercado peruano es importante trabajar constantemente en la diferenciación para siempre estar posicionados en la mente del cliente.

\section{CONCLUSIONES}

Después de realizado el análisis y la discusión sobre los resultados de las estrategias de marketing en el canal intermedio y el posicionamiento de las empresas que brindan servicio de GPS, se obtuvieron las siguientes conclusiones:

La investigación nos indicó que existe una alta relación muy significativa entre el posicionamiento y las estrategias de marketing debido a que la prueba Chi cuadrado reflejó nivel de significación 0.00 que está por debajo del crítico existiendo alta significación. Por tanto, en el análisis realizado el $69.6 \%$ de personas encuestadas, indicó que las estrategias de marketing se encuentran en la categoría Muy bueno, y donde el $97.4 \%$ se encuentra calificado como posicionamiento Muy bueno. Es fundamental la generación de estrategias de marketing eficientes para mejorar en el corto plazo el posicionamiento de las empresas comercializadoras de GPS.

La investigación nos indicó que existe una alta relación muy significativa entre el posicionamiento y los costos reducidos. La prueba $\mathrm{Chi}$ cuadrado reflejó nivel de significación 0.00 que 
está por debajo del crítico existiendo alta significación. Por tanto, en el análisis realizado el $42.9 \%$ de personas encuestadas, indicó que la estrategia de costos reducidos se encuentra en la categoría Bueno, y donde el $63 \%$ se encuentra calificado como posicionamiento Bueno.

La investigación nos indicó que existe una alta relación muy significativa entre el posicionamiento y la experiencia única al consumidor. La prueba Chi cuadrado reflejó nivel de significación 0.00 que está por debajo del crítico existiendo alta significación. Por tanto, en el análisis realizado el $38.7 \%$ de personas encuestadas, indicó que las estrategias de experiencia única al consumidor se encuentran en la categoría Muy bueno, y donde el $97.4 \%$ se encuentra calificado como posicionamiento Muy bueno.

La investigación nos indicó que existe una alta relación muy significativa entre el posicionamiento y la reinvención del modelo de negocio. La prueba Chi cuadrado reflejó nivel de significación 0.00 que está por debajo del crítico existiendo alta significación. Mientras que, en el análisis realizado el $49.4 \%$ de personas encuestadas, indicó que las estrategias reinvención del modelo de negocio se encuentran en la categoría Bueno, y donde el $75 \%$ se encuentra calificado como posicionamiento Bueno.

La investigación nos indicó que existe una alta relación muy significativa entre el posicionamiento y la calidad del producto. La prueba Chi cuadrado reflejó nivel de significación 0.00 que está por debajo del crítico existiendo alta significación. Mientras que, en el análisis realizado el $45.8 \%$ de personas encuestadas, indicó que las estrategias de calidad del producto se encuentran en la categoría Bueno, y donde el $69.4 \%$ se encuentra calificado como posicionamiento Bueno.

La investigación nos indicó que existe una alta relación muy significativa entre el posicionamiento y nicho de mercado. La prueba Chi cuadrado reflejó nivel de significación 0.00 que está por debajo del crítico existiendo alta significación. Mientras que, en el análisis realizado el $38.1 \%$ de personas encuestadas, indicó que las estrategias de nicho de mercado se encuentran en la categoría Bueno, y donde el 75.9\% se encuentra calificado como posicionamiento Bueno.
La investigación nos indicó que existe una alta relación muy significativa entre el posicionamiento y la innovación. La prueba Chi cuadrado reflejó nivel de significación 0.00 que está por debajo del crítico existiendo alta significación. Mientras que en el análisis realizado el $45.8 \%$ de personas encuestadas, indicó que las estrategias de innovación se encuentran en la categoría Bueno, y donde el $69.4 \%$ se encuentra calificado como posicionamiento Bueno.

La investigación nos indicó que existe una alta relación muy significativa entre el posicionamiento y la mejora en el diseño. La prueba Chi cuadrado reflejó nivel de significación 0.00 que está por debajo del crítico existiendo alta significación. Mientras que, en el análisis realizado el $61.3 \%$ de personas encuestadas, indicó que las estrategias de mejora en el diseño se encuentran en la categoría Bueno, y donde el 94.4\% se encuentra calificado como posicionamiento Bueno.

Finalmente, las empresas comercializadoras de GPS tendrán que aplicar eficientes estrategias de marketing en el canal intermedio (aseguradoras) con el objetivo de maximizar su rentabilidad, debido a que es un canal de crecimiento constante y rápido que garantizará su mejor posicionamiento en el mercado. Así mismo, las aseguradoras vehiculares obtienen mayores niveles de rentabilidad, además de poder controlar de mejor manera el número de siniestros y ver estrategias a tomar con sus clientes sobre como incentivar un mejor estilo de conducción, pudiendo recurrir a campañas, premios o concursos.

\section{REFERENCIAS BIBLIOGRÁFICAS}

Asociación Automotriz del Perú (2017). Venta e Inmatriculación de Vehículos Nuevos. https://aap. org.pe/estadisticas/ventas_inmatriculaciones_ vehiculos_nuevos/inm-2017/

Bocanegra Ureta, R. (2012). Desarrollo de una Aplicación Web para el monitoreo de vehículos con dispositivos GPS que comercializa una empresa de Telecomunicaciones. Universidad Ricardo Palma

Cubas, J.; Ingar, L. y Gonzales, M. (2015). Plan de Marketing para incrementar el Market Share en Servicio Prepago de Entel en el mercado de Millenials. Universidad Peruana Ciencias Aplicadas (UPC). 
Gestión (2016, 28 de noviembre). Tracklink. Estas son las conductas riesgosas que tienen los peruanos al volante. Gestión. https://gestion.pe/ tendencias/tracklink-son-conductas-riesgosas-peruanos-volante-122027-noticia/

Gestión (2017, 07 de marzo). Pacífico busca reducir accidentes de tránsito hasta en 10\% con GPS. Gestión. https://gestion.pe/economia/empresas/pacifico-busca-reducir-accidentes-transito-10-gps-smart-130160-noticia/

Kotler, P., y Armstrong, G. (2007). Marketing. México: Pearson Educación.

Kotler, P., y Keller, K. (2006). Dirección de Marketing. México: Pearson Educación.

Lamb, C., Hair, J., y McDaniel, C. (2017). Márketing. Edición Latinoamérica. México: Cengage.

Lambin, J., Gallucci, C., y Sicurello, C. (2009). Dirección de marketing. Gestión estratégica y operativa del mercado. México: McGraw Hill.

La República (2017, 09 de marzo). Pacífico Seguros presenta campaña Maneja bien y gana. $L a$ República. https://larepublica.pe/marketing/855256-pacifico-seguros-presenta-su-campana-maneja-bien-y-gana/
Lovelock, C. y Wirts, J. (2009). Marketing de servicios. Personal, tecnología y estrategia (6ta ed.). México: Pearson Educación.

McCarthy, Perreault y Perreault, William D. (2000). Marketing: un enfoque global (13va ed.). México: McGraw Hill Interamericana.

Merino Nuñez, M. y Espino Vargas, P. (2009). Posicionamiento. Chiclayo, Peru: Chiclayo In

Ministerio de Transporte y Comunicaciones. Manual de seguridad vial. Diciembre 2017. R.D. $N^{\circ} 05$ 2017-MTC/14.

Perú21 (2017, 15 de agosto). 08 de cada 10 conductores peruanos manejan agresivamente. Perú21. https://peru21.pe/lima/8-10-conductores-peruanos-manejan-agresivamente-239572-noticia/

Rivas, P. (2015, 1 de agosto). Agresividad al volante es una de las causas de accidentes. El Comercio Perú. https://elcomercio.pe/lima/agresividad-volante-causas-accidentes-188263-noticia/ 